\title{
Redaktionelle Notizen
}

Die Herzog August Bibliothek in Wolfenbüttel mit ihren reichen Quellenbeständen zur europäischen Kulturgeschichte der frühen Neuzeit hat Dank der Förderung der Stiftung Volkswagenwerk in Hannover die Möglichkeit, an inund ausländische Wissenschaftler Forschungsstipendien zu vergeben. Die Themenbereiche umfassen speziell die Wissenschafts- und Literaturgeschichte, Ideen-, Sozial- und Technikgeschichte des I6. bis I8. Jahrhunderts, für welche die Bestände der Herzog August Bibliothek in besonderer Weise nützlich sein können.

Die Stipendien sollen Wissenschaftlern aus dem In- und Ausland die Möglichkeit geben, ein Forschungsvorhaben in Wolfenbüttel zu beginnen, fortzuführen oder abzuschließen.

Die Vergabe des Wolfenbüttel-Stipendiums setzt voraus, daß der Bewerber sich durch Habilitation, Promotion oder eine vergleichbare wissenschaftliche Leistung ausgewiesen hat.

Zur Durchführung des Programms wurde im nahegelegenen Anna-VorwerkHaus eine Geschäftsstelle eingerichtet, die die persönliche Betreuung der Stipendiaten übernimmt. In dem Haus befinden sich auch Gesellschaftsräume, die für die Kommunikation unter den Wissenschaftletn bestimmt sind. Die Geschäftsstelle sorgt für die Unterbringung der Stipendiaten.

Nähere Information erteilt

die Geschäftstelle für das Stipendien- und Symposienprogramm der Herzog August Bibliothek (Leiterin Frau Dr. Sabine Solf) 334 Wolfenbüttel, Postfach 227, Tel. 0533I-22561

Der Nachlaß Robert Musils wurde im Jahr 1972 von der Republik Ósterreich erworben und der Osterreichischen Nationalbibliothek zur Restauration und Verwahrung übergeben. I973 wurde mit Unterstützung des Bundesministeriums für Wissenschaft und Forschung und des Fonds zur Förderung der wissenschaftlichen Forschung in Österreich eine Arbeitsstelle aufgebaut, die mit der Erarbeitung eines methodischen Konzepts zur Nachlaßordnung und mit der Durchführung dieser Ordnung beauftragt wurde. Sie steht unter der Leitung von Prof. Friedbert Aspetsberger und wird von Dr. Elisabeth Castex hauptamtlich betreut. Die Nachlaßmanuskripte werden mit Hilfe eines maschinenunterstützten Dokumentationssystems nach formalen und inhaltlichen Kategorien beschrieben und sortiert. Das Ziel dieser Arbeit ist die Erstellung von Katalogen und Registern zum gesamten Nachlaß, die ihn fürEditionsvorhaben und andere Forschungsprojekte aufbereiten. Die Arbeitsstelle ist auch bemüht, wissenschaftliche Arbeiten, die sich mit dem Nachlaß Robert Musils beschäftigen, fachlich zu unterstützen.

Arbeitsstelle Robert-Musil-Nachlaß

Gumpendorferstraße I $5 / \mathbf{r}$

A-1060 Wien 